\title{
Tratamiento quirúrgico del Ductus Arterioso Persistente
}

\author{
Dr. Pedro Becker R. \\ Profesor Asociado de Cirugía Cardiovascular \\ Facultad de Medicina, P. Universidad Católica de Chile \\ Recibido el 2 de octubre de 2009, aceptado el 10 de noviembre de 2009
}

Rev Chil Cardiol 2009; 28: 401-402

El Ductus Arterioso Persistente (DAP) es una cardiopatía congénita frecuente, caracterizada por cortocircuito de izquierda a derecha, que según su magnitud puede llegar a provocar insuficiencia cardíaca. Si el problema persiste por tiempo prolongado o no se resuelve oportunamente, el paciente está en riesgo de morir por complicaciones asociadas a la insuficiencia cardíaca o de desarrollar enfermedad vascular pulmonar irreversible (síndrome de Eisenmenger). En el caso de los recién nacidos de pretérmino, alrededor del $25 \%$ son portadores de un DAP, lo cual aumenta su morbimortalidad.

El cierre quirúrgico del DAP está indicado cuando éste genera un cortocircuito significativo, lo cual se manifiesta por la dilatación de las cavidades cardíacas izquierdas (aunque sea leve) asociada o no la presencia de síntomas de insuficiencia cardíaca. En los prematuros cerca del $75 \%$ de los casos responden al uso de antiprostaglandínicos, quedando un $25 \%$ restante que necesita de cierre quirúrgico.

La operación de cierre de DAP es altamente efectiva y permite resolver adecuadamente un problema potencialmente grave, dejando al paciente por lo general sin secuelas y curado de su enfermedad. Comparada con otras operaciones de cardiopatías congénitas, la del DAP es de bastante menor dificultad. Sin embargo, adquiere mayor complejidad cuando se trata de niños prematuros, principalmente los de muy bajo peso (menor a 1000 grs.) y más aún, cuando esta intervención es realizada en Centros que no cuentan con un programa de cirugía cardíaca.

En el presente número de la Revista Chilena de Cardiología se publica el trabajo "Importancia del manejo del ductus arterioso persistente en la XII región al implementar el diagnóstico ecográfico y tratamiento quirúrgico a nivel local", realizado en el Hospital Regional de Punta Arenas (agregar la referencia). En un período de 8 años se operaron 26 niños, de los cuales 16 corresponden a prematuros y 6 de ellos con peso inferior a 1000 grs. En el grupo de los prematuros hubo un fallecido por sepsis, mientras que no hubo mortalidad en el grupo de los pacientes de término. La morbilidad fue escasa y por lo tanto se puede afirmar que se trata de una experiencia con buenos resultados, si bien poco numerosa pero que cobra mayor valor al haberse desarrollado en un lugar como Punta Arenas, obedeciendo a un plan de desarrollo y asistencia por profesionales más experimentados en el diagnóstico y manejo de esta cardiopatía.

Desde el punto de vista técnico vale la pena clarificar que cuando se usa la técnica extrapleural no está indicado el uso de drenajes por cuanto no hay pneumotórax potencial que drenar, y cuando la técnica es a través de la pleura (la que personalmente prefiero por su mayor simplicidad) también puede evitarse el uso de un drenaje si se busca un mayor confort del paciente en el postoperatorio, sobretodo en niños más grandes. En cuanto a la técnica preferida para ocluir el ductus, existen distintas alternativas como la sección y sutura, la ligadura y la oclusión con hemoclip. La primera puede reservarse para pacientes con ductus muy amplios y requiere destreza en suturas vasculares. La ligadura es la técnica empleada en la presente serie, cuya ventaja principal es la mayor simplicidad, si bien tiene como desventaja la opción de dejar filtración residual.

Esto último puede aminorarse al emplear doble

Correspondencia: Dr. Pedro Becker R.

Hospital Clínico Pontificia Universidad Católica

Cirugía Cardiovascular

Correo Eléctronico:pbecker@med.puc.cl 


\section{P. Becker}

o triple ligadura, como se hizo en estos pacientes. El uso de hemoclip es en mi opinión la mejor alternativa, principalmente en los prematuros. En este grupo de pacientes el ductus puede ser friable y al disecarlo circunferencialmente y ocluirlo con una ligadura existe el riesgo de rebanarlo y romperlo. Entre más ligaduras se pongan tratando de minimizar el riesgo de ductus permeable residual, aumenta el riesgo de romperlo con la consecuente hemorragia que puede ser difícil de controlar y por ende potencialmente letal. EI hemoclip en cambio no requiere de disección circunferencial del ductus y lo ocluye en forma pareja y controlada, tal como lo haría un clamp vascular. Pienso que es útil que los cirujanos enfrentados a estos pacientes conozcan y manejen hábilmente estas distintas alternativas y por lo tanto debe estar dentro de los objetivos del entrenamiento de médicos locales dispuestos a hacer estas operaciones.

En los grupos cardioquirúrgicos desarrollados, incluídos los chilenos, el DAP es actualmente tratado mediante cierre percutáneo en la mayoría de los pacientes con peso superior a los $6 \mathrm{~kg}$., quedando el cierre quirúrgico reservado principalmente para los prematuros y casos especiales que no sean candidatos a cierre percutáneo, principalmente por razones anatómicas. En la Universidad Católica el mayor volumen de cirugía ductal durante la presente década corresponde a prematuros. En la experiencia más recientemente reportada durante el II Congreso de Chileno de Neonatología, 52 recién nacidos de pre-término fueron operados entre los años 2000 a 2006. Su edad gestacional promedio fue $26,3 \pm 1,9$ semanas y su peso promedio de $891 \pm 289$ grs. Los pacientes se operaron en promedio a los $22 \pm 13$ días utilizando la vía transpleural y empleando hemoclip de titanio para su oclusión.

Todas las intervenciones se realizaron en la unidad de Neonatología para minimizar las chances de problemas asociados al traslado de estos niños. La complicación más frecuente fue la hipotensión en el $30 \%$, seguida de atelectasias en el $11 \%$ y sepsis en el $7 \%$ de los casos. Los días de ventilación mecánica postoperatoria tuvieron una mediana de 4 (1 a 67) y los días de hospitalización fueron 105 (44 a 194). No hubo mortalidad a los 30 días y un paciente falleció alejadamente.

La cirugía del ductus en general no constituye una urgencia médica y por lo tanto siempre existe la disponibilidad de tiempo para efectuar un traslado, incluyendo los prematuros. Sin embargo es efectivo que trasladar niños desde lugares alejados implica costos económicos y también algún grado de riesgo, principalmente en los prematuros extremos. Es aquí donde se debe sopesar las ventajas y desventajas de operar un niño en su hospital de origen versus trasladarlo a un centro con mayor experiencia, incluyendo en la ecuación la incidencia local de pacientes con DAP.

Creo que un paso inicial muy importante ha sido dado por el grupo de Punta Arenas al mejorar su proceso diagnóstico con ayuda de la telemedicina y un Hospital de mucha experiencia, que además ha contribuido a entrenar un neonatólogo en el diagnóstico ecocardiográfico de ductus. El modelo de llevar un cirujano experimentado me parece correcto, pero sólo para una etapa inicial. El modelo ideal en mi opinión, es que Punta Arenas llegue a contar con un cardiólogo pediatra que permita precisión y agudeza diagnóstica y con un cirujano local bien entrenado, competente y autovalente. $Y$ a esto le agregaría volumen de actividad, ya que 2 o 3 casos anuales hacen más difícil adquirir adecuada experiencia.

Una forma de adquirir mayor volumen es que grupos como el de Punta Arenas concentren la actividad de una parte del país. No creo que sea buena idea establecer muchos centros dispersados por todo el territorio en que cada uno haga un volumen pequeño de cirugía ductal.

El grupo de los niños prematuros extremos (menor a $1000 \mathrm{gr}$ ) constituye un grupo especialmente grave, en los que podría argumentarse que su mejor opción de tratamiento es en un centro de alto volumen y nivel de complejidad. Sin embargo, la experiencia reportada que motiva esta editorial, si bien es aún pequeña, exhibe muy buenos resultados, incluidos los pocos casos de prematuros extremos.

Creo que será de mucho interés conocer en el futuro cómo eventualmente ha progresado y se ha sostenido esta experiencia en el tiempo, por lo que estimulo a los autores a reportar sus resultados en algunos años más y a dar los pasos siguientes para constituirse en un grupo autónomo. 